\title{
Synthesis and Biological Evaluation of Novel Triazolyl-Acridine Derivatives as Cytotoxic Agents
}

\author{
M. SINGH* D. N PRASAD ${ }^{1}$ AND SUPRIYA AGNIHOTRI ${ }^{2}$ \\ Research Scholar, IKG Punjab Technical University, Kapurthala, Jalandhar-144 603, ${ }^{1}$ Shivalik College of Pharmacy, \\ Nangal-140 126, ${ }^{2}$ Chandigarh College of Pharmacy, Landran, Punjab-140307, India
}

Singh et al.: Synthesis of Novel Triazolyl-Acridine derivatives as Cytotoxic Agents

\begin{abstract}
Novel triazolyl-acridine compounds were synthesized in 4 series of 9-(2-(substituted phenyl-1H1,2,3-triazol-1-yl)ethoxy)acridine, 9-(3-(4-(2-substituted phenyl)-1H-1,2,3-triazol-1-yl)propoxy) acridine, N-(2-(4 substituted phenyl-1H-1,2,3-triazol-1-yl)ethyl)acridin-9-amine and N-(3(4-(substituted phenyl)-1H-1,2,3-triazol-1-yl)propyl)acridin-9-amine using appropriate synthetic procedures and screened for cytotoxic activity. The structures of all synthesized compounds were confirmed by Fourier-transform infrared spectroscopy, proton nuclear magnetic resonance and mass spectroscopy and these compounds were assayed in vitro for cytotoxic activity against MCF-7 (human breast adenocarcinoma cell line) and HT-29 (human colon adenocarcinoma cell line) cells. Tested compounds showed better cytotoxic activities in terms of $\mathrm{IC}_{50}$ value against MCF-7 and HT-29 cells. Methyl substituted compound MPP-9 exhibited excellent sensitivity with $\mathrm{IC}_{50}$ value 1 and $2 \mu \mathrm{M}$, against MCF-7 and HT-29, respectively. Unsubstituted MPP-1 and chloro-substituted MPP-2 and MPP-5 also exhibited good $\mathrm{IC}_{50}$ value ranges from 2-4 $\mu \mathrm{M}$ against both cell lines. These compounds were active at micro molar concentrations. Data study revealed that synthesized compounds are promising leads for future as cytotoxic agents.
\end{abstract}

Key words: Acridine, Triazol, Cytotoxic, Cell line

Without a doubt, no one deny the fact that cancer is an epidemic greater than one of the biggest medical challenges of the century ${ }^{[1]}$. There are many types of cancer e.g. breast cancer, colon cancer, lung cancer, prostate cancer, but all types of cancer have similarity of uncontrolled proliferation or uncontrolled symmetric cell division ${ }^{[2]}$. Technologies for diagnosis and treatment are highly advanced now a day, but the treatment still remains poor.

Literature survey revealed many heterocyclic organic compounds like acridines, triazoles, which are effective against cancer. Acridine is a tricycle organic compound with a nitrogen heterocycle (fig. 1) having molecular formulae $\mathrm{C}_{13} \mathrm{H}_{9} \mathrm{~N}$, which can also be named as 10-azaanthracene and dibenzo[b,e]pyridine ${ }^{[3]}$. Inhibitory activity on tumor cells and binding to DNA is the popular molecular strategy. DNA targeting is a recent trend in innovation of new anticancer agents. In 1998, a quantitative structure-activity relationship (QSAR) analysis of 9-anilinoacridines with respect to

*Address for correspondence E-mail: mandeepchadha7@gmail.com July-August 2020 antitumor activity and binding to DNA was reported by Gao et al. ${ }^{[4]}$. Intercalative binding of proflavine to bacterial nucleic acids is the site of action ${ }^{[5]}$, which led to the development of acridine derivatives for modern anticancer chemotherapy e.g. m-AMSA, although the actual site of action of such derivatives is now established at the level of DNA coiling enzymes (topoisomerase) rather than DNA itself, damage being caused by the stabilization of the enzyme-DNA cleavage complex ${ }^{[6,7]}$. Acridine derivatives are not only effective antitumor ${ }^{[8]}$ but also effective antiinflammatory ${ }^{[9]}$, antibacterial ${ }^{[10]}$, antimalarial $^{[11]}$ and antiviral agents ${ }^{[12]}$.

Triazoles are heterocyclic organic compounds containing five-membered ring with 3 nitrogen and This is an open access article distributed under the terms of the Creative
Commons Attribution-NonCommercial-ShareAlike 3.0 License, which
allows others to remix, tweak, and build upon the work non-commercially,
as long as the author is credited and the new creations are licensed under
the identical terms

Accepted 17 July 2020

Revised 11 May 2020

Received 28 January 2020 Indian J Pharm Sci 2020;82(4):586-592 


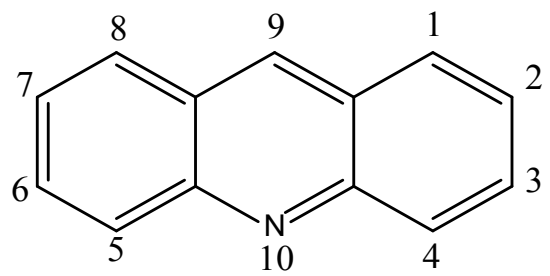

Fig. 1: Structure of acridine

2 carbon atoms. Two isomeric forms of triazoles exist namely 1,2,3-triazole and 1,2,4-triazole ${ }^{[13]}$, with molecular formula $\mathrm{C}_{2} \mathrm{H}_{3} \mathrm{~N}_{3}, 1,2,3$-triazole is a basic aromatic heterocycle. The medicinal chemists have considered the synthesis of 1,2,3-triazole based heterocycles as the corner stone of medicinal chemistry due to their important biological activities like anticancer ${ }^{[14]}$, antitubercular ${ }^{[15]}$, antibacterial ${ }^{[16]}$, antiinflammatory ${ }^{[17]}$, antimicrobia ${ }^{[18]}$ and antiviral ${ }^{[19]}$. The features possessed by the 1,2,3-triazoles make them pharmaceutically important molecules.

Thus, both acridines and 1,2,3-triazoles are important pharmacophores with anticancer activity. Both compounds are heterocyclic, small in size, with ease of synthesis and reported to possess extensively anticancer activity. Therefore in this investigation both structures were combined to synthesize novel triazolyl-acridine derivatives for evaluating anticancer activity against different cell lines.

\section{MATERIALS AND METHODS}

Synthetic grade chemicals were used. Thin layer chromatography (TLC) on precoated TLC plates was used to monitor the synthesis and finally prepared compounds. Chloroform:methanol (9:1) was used as the mobile phase for TLC and iodine chamber to visualized spots. Open glass capillary method was used for melting point determination. Infrared (IR) spectra were recorded on Bruker's FT-IR spectrophotometer as $\mathrm{KBr}$ pellet and values are expressed in $\mathrm{cm}^{-1} .{ }^{1} \mathrm{H}$ nuclear magnetic resonance spectroscopy (NMR) spectral analysis of the synthesized compounds were recorded on a Bruker Avance II $400 \mathrm{MHz}$ in deuterated chloroform $\left(\mathrm{CDCl}_{3}\right)$ using tetramethylsilane as internal standard. Chemical shift values were recorded on $\delta$-scale. Syntheses of the triazolyl-acridine derivatives were performed according to the scheme presented in fig. 2.

\section{Synthesis of 9-chloroacridine N-phenylanthranilic acid:}

and

9-Chloroacridine was synthesized using modified Ullman-Goldberg reaction ${ }^{[20]}$. N-phenylanthranilic acid was synthesized by mixing equal quantities of $(0.038 \mathrm{~mol})$ of o-chlorobenzoic acid and aniline with $0.12 \mathrm{~g}$ of copper powder in $40 \mathrm{ml}$ isoamyl alcohol to which $6 \mathrm{~g}$ of dry potassium carbonate was added slowly and the final mixture was refluxed for 6 to $8 \mathrm{~h}$. After reflux, isoamyl alcohol was removed by distillation, the mixture poured into $500 \mathrm{ml}$ of hot water and filtered. The filtrate was acidified with concentrated hydrochloric acid. Yellow precipitate formed, filtered, washed with hot water and collected.

\section{Synthesis of 9-chloroacridineby cyclization of n-phenylanthranilic acid:}

Five grams ( $0.023 \mathrm{~mol})$ of N-phenylanthranilic acid was mixed with $16 \mathrm{ml}$ ( $27 \mathrm{~g}, 0.176$ moles) of phosphorus oxychloride taken in a $500 \mathrm{ml}$ round bottom flask fitted with a water-cooled condenser and heated about $15 \mathrm{~min}$ at $85-90^{\circ}$, then temperature is raised to $135-140^{\circ}$, where it was maintained for $2 \mathrm{~h}$. Excess phosphorus oxychloride was removed by simple distillation. After cooling, the residue was poured into a well-stirred mixture of $20 \mathrm{ml}$ of concentrated ammonia solution, $50 \mathrm{~g}$ of ice and $20 \mathrm{ml}$ of chloroform in a separating funnel and allowed to stand for 30-40 min. The chloroform layer was separated and evaporated, greenish gray powder obtained was 9-chloroacridine.

\section{Addition of alkanediol to 9-chloroacridine ${ }^{[21]}$ :}

Dissolved $0.2 \mathrm{~g}(1.0 \mathrm{mmol})$ of 9-chloroacridine in $2.0 \mathrm{ml}$ of ethylene glycol, under inert atmosphere, a $1.0 \mathrm{M}$ solution of potassium t-butoxide in t-butyl alcohol (1.5 ml, $1.5 \mathrm{mmol}, 1.5$ equiv) was added, stirred at $80^{\circ}$ for $18 \mathrm{~h}$, then quenched with saturated $\mathrm{NaHCO}_{3}$. The mixture was extracted with $\mathrm{CH}_{2} \mathrm{Cl}_{2}$, dried over anhydrous $\mathrm{MgSO}_{4}$, concentrated in vacuum, and purified by recrystallization from $\mathrm{CHCl}_{3}$ to yield 9-acridinyl alkanediol as the product.

\section{Conversion of 9-chloroacridine to amino alcohols:}

9-chloroacridine $(1.0 \mathrm{~g}, 5.1 \mathrm{mmol})$ and alkanolamine (5 equiv) was heated at $110^{\circ}$ for $6 \mathrm{~h}$, under inert atmosphere and then cooled. $\mathrm{NaOH} 1 \mathrm{~N}$ was added and extracted with $\mathrm{CHCl}_{3}$. The organic layers were washed with brine, dried over anhydrous $\mathrm{Na}_{2} \mathrm{SO}_{4}$ and evaporated under reduced pressure to yield 9-acridinyl alkanolamine as the product.

\section{Conversion of alcohols into azides $^{[22]}$ :}

Firstly, 3 mmol of 9-acridinyl alkanediol or 9-acridinyl alkanolamine, $3.6 \mathrm{mmol}$ of triphenylphosphine and 
<smiles>O=C(O)c1ccccc1Cl</smiles><smiles>Nc1ccccc1</smiles><smiles>O=C(O)c1ccccc1Nc1ccccc1</smiles>

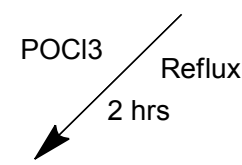<smiles>Clc1c2ccccc2nc2ccccc12</smiles>

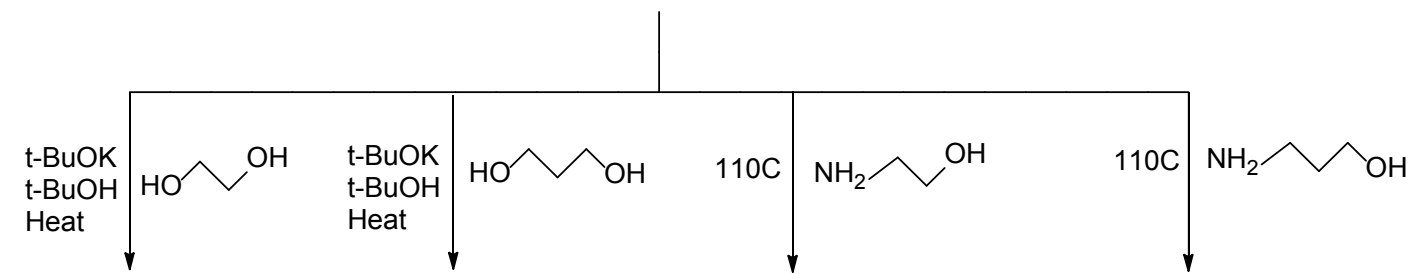<smiles>OCCOc1c2ccccc2nc2ccccc12</smiles><smiles>OCCCOc1c2ccccc2nc2ccccc12</smiles><smiles>OCCNc1c2ccccc2nc2ccccc12</smiles><smiles>OCCCNc1c2ccccc2nc2ccccc12</smiles>

\begin{tabular}{l|} 
PPh3 \\
12 \\
NaN3 \\
DMSO
\end{tabular}<smiles>NCCO[Te]</smiles><smiles>NCCCOc1c2ccccc2nc2ccccc12</smiles>

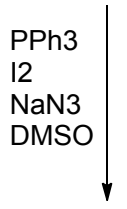<smiles>c1ccc2nc3ccccc3cc2c1</smiles><smiles>N#CCNc1c2ccccc2nc2ccccc12</smiles><smiles>Cc1ccccn1</smiles>

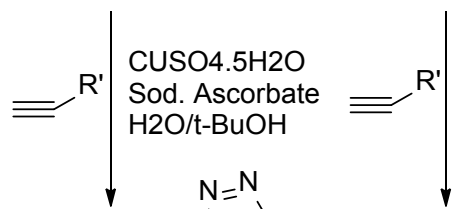<smiles>[R]c1cn(CCOc2c3ccccc3nc3ccccc23)nn1</smiles><smiles>[R]c1cn(CCCOc2c3ccccc3nc3ccccc23)nn1</smiles><smiles>[R]c1cn(CCNc2c3ccccc3nc3ccccc23)nn1</smiles><smiles>N#CCCNc1c2ccccc2nc2ccccc12</smiles><smiles>[R]=[Fe]</smiles><smiles>C#Cc1ccccc1Cl</smiles><smiles>CC#Cc1ccccc1</smiles>

Fig. 2: Synthetic scheme to prepare target compounds MPP-1 to MPP-12

$3.6 \mathrm{mmol}$ of iodine was triturated in a mortar and pestle for $10 \mathrm{~min}$, when exothermic reaction took place, paste like consistency appeared. Separately, $12 \mathrm{mmol}$ of sodium azide was dissolved in DMSO, the solution was mixed with acridine paste and stirred for 30 min on a magnetic stirrer. Upon completion of the reaction, ice- cooled solution of $50 \mathrm{ml}$ of sodium thiosulphate was added and extracted with $30 \mathrm{ml}$ of diethyl ether 3 times. The combined organic layer was washed with brine $(50 \mathrm{ml})$. On evaporation a crude product obtained, which was purified by column chromatography using $5 \%$ ethyl acetate in hexane. 


\section{Conversion of azides in to triazoles ${ }^{[23]}$ :}

Three mmol of alkyne and, $3 \mathrm{mmol}$ of azide were suspended in $12 \mathrm{ml}$ of a 1:1 water/tert-butanol mixture. Freshly prepared $1 \mathrm{M}$ sodium ascorbate solution was added, followed by $0.03 \mathrm{mmol}$ of copper (II) sulfate pentahydrate in $100 \mathrm{ml}$ of water. The heterogeneous mixture was stirred vigorously overnight. When TLC analysis indicated complete consumption of the reactants, the reaction mixture was diluted with $50 \mathrm{ml}$ of water and cooled in ice, and the white precipitate was collected as filtrate. After being washed with cold water $(225 \mathrm{ml})$, the precipitate was dried under vacuum to obtain pure triazole compounds, MPP-1 to MPP-12.

MPP-1, 9-(2-(4-phenyl-1H-1,2,3-triazol-1-yl)ethoxy) acridine, yield: $85 \%$, melting point (MP): $223^{\circ}, \mathrm{R}_{\mathrm{f}}$ : 0.45;FT-IR (KBr): $\mathrm{cm}^{-1} 3051 \mathrm{C}-\mathrm{H}$ str. (triazole), 1463 $\mathrm{C}-\mathrm{H}$ str. (methylene), $1651 \mathrm{C}=\mathrm{N}$ str. (Ar), $1592 \mathrm{~N}=\mathrm{N}$ str., $1524 \mathrm{C}=\mathrm{C}$ str. (Ar), $1308 \mathrm{C}-\mathrm{N}$ str., $1254 \mathrm{C}-\mathrm{O}$ str. (ether), $\mathrm{C}-\mathrm{Cl}$ str. (Ar); ${ }^{1} \mathrm{H} \mathrm{NMR}\left(\mathrm{CDCl}_{3}, 400 \mathrm{MHz}\right): \delta$; 8.14 (d, 2H, $J=9.44, \mathrm{CH}), 7.99$ (d, 2H, $J=8.2, \mathrm{CH}$ ), 7.82 (t, 2H, $J=4.96,4.2, \mathrm{CH}), 7.79$ (d, 2H, $J=6.92$, $\mathrm{CH}), 7.63(\mathrm{t}, 2 \mathrm{H}, J=4.08,3.68, \mathrm{CH}), 7.59(\mathrm{~s}, 1 \mathrm{H}, \mathrm{CH}-$ triazole), 7.53 (t, $2 \mathrm{H}, J=2.8,2.96, \mathrm{CH}-\mathrm{Ar}), 7.45$ (t, $1 \mathrm{H}$, $J=2.48,4.72, \mathrm{CH}-\mathrm{Ar}), 4.50$ (t, $2 \mathrm{H}, J=6.56,7.32, \mathrm{CH})$, 4.16 (t, 2H, $J=5.24,5.08, \mathrm{CH}) \mathrm{ppm} ; \mathrm{MS}(\mathrm{m} / \mathrm{z}): 367$ $(\mathrm{M}+1)$.

MPP-2, 9-(2-(4-(2-chlorophenyl)-1H-1,2,3-triazol-1yl)ethoxy)acridine, yield: $83 \%$, MP: $242^{\circ}, \mathrm{R}_{\mathrm{f}}: 0.68$;FTIR (KBr): $\mathrm{cm}^{-1} 3048 \mathrm{C}-\mathrm{H}$ str. (triazole), $1466 \mathrm{C}-\mathrm{H}$ str. (methylene), $1656 \mathrm{C}=\mathrm{N}$ str. (Ar), $1541 \mathrm{~N}=\mathrm{N}$ str., 1510 $\mathrm{C}=\mathrm{C}$ str. (Ar), $1313 \mathrm{C}-\mathrm{N}$ str., $1278 \mathrm{C}-\mathrm{O}$ str. (ether), 836 $\mathrm{C}-\mathrm{Cl}$ str. (Ar); ${ }^{1} \mathrm{H}$ NMR $\left(\mathrm{CDCl}_{3}, 400 \mathrm{MHz}\right): \delta 8.12(\mathrm{~d}$, $2 \mathrm{H}, J=10.28, \mathrm{CH}), 7.94(\mathrm{~d}, 2 \mathrm{H}, J=6.8, \mathrm{CH}), 7.79$ (t, $2 \mathrm{H}, J=5.08,6.84, \mathrm{CH}-\mathrm{Ar}), 7.74$ (d, $1 \mathrm{H}, J=8.76, \mathrm{CH})$, $7.64(\mathrm{t}, 2 \mathrm{H}, J=5.24,3.6, \mathrm{CH}), 7.59$ (s, $1 \mathrm{H}, \mathrm{CH}$-triazole), 7.57 (d, $1 \mathrm{H}, J=7.84,2.96$, CH-Ar), 7.39 (t, $1 \mathrm{H}, J=4.0$, 4.2, CH-Ar), 7.35 (t, 1H, J=2.88, 6.44, CH-Ar), 4.49 (t, $2 \mathrm{H}, J=2.08,7.52, \mathrm{CH}), 4.16$ (t, 2H, $J=7.24,2.56, \mathrm{CH})$ ppm. MS (m/z); $401(\mathrm{M}+1)$.

MPP-3, 9-(2-(4-(o-tolyl)-1H-1,2,3-triazol-1-yl)ethoxy) acridine, yield: $82 \%$, MP: $212^{\circ}, \mathrm{R}_{\mathrm{f}}: 0.58$; FT-IR (KBr): $\mathrm{cm}^{-1} 3052 \mathrm{C}-\mathrm{H}$ str. (triazole), $1455 \mathrm{C}-\mathrm{H}$ str. (methylene), $1436 \mathrm{C}-\mathrm{H}$ str. (methyl), $1651 \mathrm{C}=\mathrm{N}$ str., $1524 \mathrm{~N}=\mathrm{N}$ str., $1308 \mathrm{C}-\mathrm{N}$ str., $1254 \mathrm{C}-\mathrm{O}$ str. (ether). ${ }^{1} \mathrm{H} \mathrm{NMR}\left(\mathrm{CDCl}_{3}\right.$, $400 \mathrm{MHz}): \delta 8.14(\mathrm{~d}, 2 \mathrm{H}, J=6.8, \mathrm{CH}), 7.98(\mathrm{~d}, 2 \mathrm{H}, J$ $=6.84, \mathrm{CH}), 7.83(\mathrm{t}, 2 \mathrm{H}, J=6.44,1.16, \mathrm{CH}-\mathrm{Ar}), 7.63$ $(\mathrm{t}, 2 \mathrm{H}, J=3.6,5.32, \mathrm{CH}), 7.60(\mathrm{~s}, 1 \mathrm{H}, \mathrm{Ar}-\mathrm{CH}), 7.56(\mathrm{~d}$, $1 \mathrm{H}, J=9.64, \mathrm{CH}-\mathrm{Ar}$ ), 7.33-7.27 (m, 3H, CH-Ar), 4.51 (t, 2H, $J=4.88,4.64, \mathrm{CH}), 4.15(\mathrm{t}, 2 \mathrm{H}, J=3.6,5.2, \mathrm{CH})$, $2.58\left(\mathrm{~s}, 3 \mathrm{H}, \mathrm{CH}_{3}\right) \mathrm{ppm}$. MS (m/z): $381(\mathrm{M}+1)$.

MPP-4, 9-(3-(4-phenyl-1H-1,2,3-triazol-1-yl)propoxy) acridine, yield: $90 \%$, MP: $242^{\circ}, \mathrm{R}_{\mathrm{f}}: 0.46$; FT-IR (KBr): $\mathrm{cm}^{-1}$ 3054C-H str. (triazole), $1452 \mathrm{C}-\mathrm{H}$ str. (methylene), $1654 \mathrm{C}=\mathrm{N}$ str., $1541 \mathrm{~N}=\mathrm{N}$ str., $1511 \mathrm{C}=\mathrm{C}$ str. $(\mathrm{Ar}), 1307$ $\mathrm{C}-\mathrm{N}$ str., $1259 \mathrm{C}-\mathrm{O}$ str. (ether); ${ }^{1} \mathrm{H}$ NMR $\left(\mathrm{CDCl}_{3}, 400\right.$ MHz): $\delta 8.13(\mathrm{~d}, 2 \mathrm{H}, J=9.28, \mathrm{CH}), 7.97(\mathrm{~d}, 2 \mathrm{H}, J$ $=8.84, \mathrm{CH}), 7.82(\mathrm{t}, 2 \mathrm{H}, J=1.84,6.08, \mathrm{CH}-\mathrm{Ar}), 7.79(\mathrm{~d}$, $2 \mathrm{H}, J=8.44, \mathrm{CH}), 7.63$ (t, $2 \mathrm{H}, J=4.64,6.0, \mathrm{CH}), 7.59$ (s, 1H, Ar-CH), 7.53 (t, 2H, J=4.6, 5.32, CH-Ar), 7.42 (t, $1 \mathrm{H}, J=4.12,4.6, \mathrm{CH}-\mathrm{Ar}), 4.46$ (t, 2H, $J=1.6,5.44$, $\mathrm{CH}), 4.06(\mathrm{t}, 2 \mathrm{H}, J=5.08,2.0, \mathrm{CH}), 2.21-2.17(\mathrm{p}, 2 \mathrm{H}$, $\mathrm{CH}) \mathrm{ppm}$. MS (m/z): $381(\mathrm{M}+1)$

MPP-5, 9-(3-(4-(2-chlorophenyl)-1H-1,2,3-triazol-1yl)propoxy)acridine, yield: $81 \%$, MP: $221^{\circ}, \mathrm{R}_{\mathrm{f}}: 0.43$; FT-IR (KBr): $\mathrm{cm}^{-1} 3072 \mathrm{C}-\mathrm{H}$ str. (triazole), $1436 \mathrm{C}-\mathrm{H}$ str. (methylene), $1658 \mathrm{C}=\mathrm{N}$ str., $1545 \mathrm{~N}=\mathrm{N}$ str., 1504 $\mathrm{C}=\mathrm{C}$ str.(Ar), $1309 \mathrm{C}-\mathrm{N}$ str., $1256 \mathrm{C}-\mathrm{O}$ str. (ether), 836 $\mathrm{C}-\mathrm{Cl}$ str.(Ar); ${ }^{1} \mathrm{H}$ NMR $\left(\mathrm{CDCl}_{3}, 400 \mathrm{MHz}\right): \delta 8.14$ (d, $2 \mathrm{H}, J=6.12, \mathrm{CH}), 7.99(\mathrm{~d}, 2 \mathrm{H}, J=5.12, \mathrm{CH}), 7.82(\mathrm{t}$, $2 \mathrm{H}, J=3.4,2.88, \mathrm{CH}-\mathrm{Ar}), 7.76(\mathrm{~d}, 1 \mathrm{H}, J=8.0 \mathrm{CH}), 7.63$ (t, 2H, $J=2.8,3.6, \mathrm{CH}), 7.59$ (s, 1H, Ar-CH), 7.55 (d, $1 \mathrm{H}, J=7.36, \mathrm{CH}-\mathrm{Ar}$ ), 7.39 (t, $1 \mathrm{H}, J=8.28,3.36$, CHAr), 7.35 (t, 1H, $J=5.08,3.08, \mathrm{CH}-\mathrm{Ar}), 4.48(\mathrm{t}, 2 \mathrm{H}$, $J=7.32,3.44, \mathrm{CH}), 4.07$ (t, 2H, 4.68, $J=2.84,4, \mathrm{CH})$ 2.21-2.17 (p, 2H, CH) ppm. MS (m/z): $415(\mathrm{M}+1)$.

MPP-6, 9-(3-(4-(o-tolyl)-1H-1,2,3-triazol-1-yl) propoxy)acridine: yield, $79 \%$, MP: $215^{\circ} \mathrm{C}, \mathrm{R}_{\mathrm{f}}: 0.61$; FT-IR (KBr): $\mathrm{cm}^{-1} 3073 \mathrm{C}-\mathrm{H}$ str. (triazole), $1468 \mathrm{C}-\mathrm{H}$ str. (methylene), $1438 \mathrm{C}-\mathrm{H}$ str. (methyl), $1652 \mathrm{C}=\mathrm{N}$ str., $1546 \mathrm{~N}=\mathrm{N}$ str., $1507 \mathrm{C}=\mathrm{C}$ str. (Ar), $1313 \mathrm{C}-\mathrm{N}$ str., $1255 \mathrm{C}-\mathrm{O}$ str. (ether); ${ }^{1} \mathrm{H}$ NMR $\left(\mathrm{CDCl}_{3}, 400 \mathrm{MHz}\right): \delta$ $8.14(\mathrm{~d}, 2 \mathrm{H}, J=6.8, \mathrm{CH}), 7.98(\mathrm{~d}, 2 \mathrm{H}, J=9.84, \mathrm{CH})$, 7.81 (t, 2H, $J=2.36,3.6, \mathrm{CH}-\mathrm{Ar}), 7.62$ (t, 2H, $J=7.08$, 2.48, CH), 7.59 (s, $1 \mathrm{H}, \mathrm{Ar}-\mathrm{CH}), 7.56(\mathrm{~d}, 1 \mathrm{H}, J=10.04$, $\mathrm{CH}), 7.35$ (t, $1 \mathrm{H}, J=2.88,6.44, \mathrm{CH}-\mathrm{Ar}), 7.30-7.26$ (m, $2 \mathrm{H}, \mathrm{CH}-\mathrm{Ar}), 4.48$ (t, 2H, $J=4.2,5.16, \mathrm{CH}), 4.09$ (t, 2H, $J=6.4,3.96, \mathrm{CH}), 2.59$ (s, 3H, $\left.\mathrm{CH}_{3}\right), 2.21-2.17$ (p, 2H, $\mathrm{CH}) \mathrm{ppm}$. MS (m/z): $395(\mathrm{M}+1)$.

MPP-7, N-(2-(4-phenyl-1H-1,2,3-triazol-1-yl)ethyl) acridin-9-amine: yield, $82 \%$, MP: $232^{\circ}, \mathrm{R}_{\mathrm{f}}$ : 0.49; FTIR (KBr): $\mathrm{cm}^{-1} 3339 \mathrm{~N}-\mathrm{H}$ str. (secondary amine), 3075 $\mathrm{C}-\mathrm{H}$ str. (triazole), $1468 \mathrm{C}-\mathrm{H}$ str. (methylene), $1655 \mathrm{C}=\mathrm{N}$ str., $1545 \mathrm{~N}=\mathrm{N}$ str., $1510 \mathrm{C}=\mathrm{C}$ str. $(\mathrm{Ar}), 1311 \mathrm{C}-\mathrm{N}$ str.; ${ }^{1} \mathrm{H}$ $\mathrm{NMR}\left(\mathrm{CDCl}_{3}, 400 \mathrm{MHz}\right): \delta 8.18(\mathrm{~d}, 2 \mathrm{H}, J=7.44, \mathrm{CH})$, 7.98 (d, 2H, $J=6.8, \mathrm{CH}), 7.81(\mathrm{~d}, 2 \mathrm{H}, J=8.64, \mathrm{CH})$, 7.78 (t, 2H, $J=2.56,4.44$, CH-Ar), 7.62 (t, 2H, $J=5.24$, 5.08, CH), 7.59 (s, 1H, Ar-CH), 7.52 (t, 2H, $J=3.24$, 
7.2, CH-Ar), 7.42 (t, 1H, $J=5.36,3.36, \mathrm{CH}-\mathrm{Ar}$ ), 5.61 (t, 2H, $J=4.2,4.8, \mathrm{CH}), 4.01$ (s, 1H, Ar-NH), 3.56 (t, 2H, $J=4.56,6.48, \mathrm{CH}) \mathrm{ppm}$. MS (m/z): $366(\mathrm{M}+1)$.

MPP-8, N-(2-(4-(2-chlorophenyl)-1H-1,2,3-triazol1-yl) ethyl)acridin-9-amine, yield: $85 \%$, MP: $236^{\circ}$, $\mathrm{R}_{\mathrm{f}}$ : 0.54; FT-IR (KBr): $\mathrm{cm}^{-1} 3334-\mathrm{N}-\mathrm{H}$ str. (secondary amine), 3061-C-H str. (triazole), 1452-C-H str. (methylene), $1650-\mathrm{C}=\mathrm{N}$ str., $1545-\mathrm{N}=\mathrm{N}$ str., $1505-\mathrm{C}=\mathrm{C}$ str. (Ar), $1310 \mathrm{C}-\mathrm{N}$ str., $832 \mathrm{C}-\mathrm{Cl}$ str.; ${ }^{1} \mathrm{H}$ NMR $\left(\mathrm{CDCl}_{3}\right.$, $400 \mathrm{MHz}$ ): $\delta 8.19$ (d, 2H, $J=8.24, \mathrm{CH}), 7.95$ (d, 2H, $J$ $=7.52, \mathrm{CH}), 7.78(\mathrm{t}, 2 \mathrm{H}, J=1.32,5.32, \mathrm{CH}-\mathrm{Ar}), 7.74(\mathrm{~d}$, $1 \mathrm{H}, J=8.76, \mathrm{CH}), 7.61$ (t, 2H, $J=1.64,3.92, \mathrm{CH}), 7.59$ (s, 1H, Ar-CH), 7.55 (d, 1H, $J=9.32, \mathrm{CH}-\mathrm{Ar}), 7.40-$ 7.35 (m, 2H, CH-Ar), 5.60 (t, 2H, $J=3.88,3.36, \mathrm{CH}$ ), $4.00(\mathrm{~s}, 1 \mathrm{H}, \mathrm{NH}), 3.56(\mathrm{t}, 2 \mathrm{H}, J=2.12,4.92, \mathrm{CH}) \mathrm{ppm}$. $\mathrm{MS}(\mathrm{m} / \mathrm{z}): 400(\mathrm{M}+1)$.

MPP-9, N-(2-(4-(o-tolyl)-1H-1,2,3-triazol-1-yl)ethyl) acridin-9-amine: yield, $86 \%$, MP: $227^{\circ}, \mathrm{R}_{\mathrm{f}}$ : 0.62; FT-IR (KBr): $\mathrm{cm}^{-1} 3379-\mathrm{N}-\mathrm{H}$ str. (secondary amine), 3069-C$\mathrm{H}$ str. (triazole), 1475-C-H str. (methylene), 1435-C-H str. (methyl), $1655-\mathrm{C}=\mathrm{N}$ str., $1590-\mathrm{N}=\mathrm{N}$ str., $1538-\mathrm{C}=\mathrm{C}$ str. (Ar), 1308-C-N str.; ${ }^{1} \mathrm{H}$ NMR $\left(\mathrm{CDCl}_{3}, 400 \mathrm{MHz}\right)$ : $\delta 8.20(\mathrm{~d}, 2 \mathrm{H}, J=11.12, \mathrm{CH}), 7.96(\mathrm{~d}, 2 \mathrm{H}, J=7.32$, $\mathrm{CH}), 7.78$ (t, 2H, $J=1.36,4.72, \mathrm{CH}-\mathrm{Ar}), 7.62(\mathrm{t}, 2 \mathrm{H}$, $J=3.04,5.24, \mathrm{CH}), 7.59$ (s, 1H, Ar-CH), 7.57 (d, 1H, $J=10.28$, CH-Ar), 7.33-7.28 (m, 3H, CH-Ar), 5.59 (t, $2 \mathrm{H}, J=3.36,4.96, \mathrm{CH}), 4.01(\mathrm{~s}, 1 \mathrm{H}, \mathrm{NH}), 3.56(\mathrm{t}, 2 \mathrm{H}$, $J=3.24,3.2, \mathrm{CH}), 2.59$ (s, $\left.3 \mathrm{H}, \mathrm{CH}_{3}\right) \mathrm{ppm}$. MS (m/z): $380(\mathrm{M}+1)$.

MPP-10, N-(3-(4-phenyl-1H-1,2,3-triazol-1-yl)propyl) acridin-9-amine, yield: $89 \%$, MP: $217^{\circ}, \mathrm{R}_{\mathrm{f}}$ : 0.67; FTIR (KBr): $\mathrm{cm}^{-1} 3365-\mathrm{N}-\mathrm{H}$ str. (secondary amine), 3078$\mathrm{C}-\mathrm{H}$ str. (triazole), 1457-C-H str. (methylene), 1656$\mathrm{C}=\mathrm{N}$ str., $1597-\mathrm{N}=\mathrm{N}$ str., $1535-\mathrm{C}=\mathrm{C}$ str. $(\mathrm{Ar}), 1302-$ $\mathrm{C}-\mathrm{N}$ str.; ${ }^{1} \mathrm{H}$ NMR $\left(\mathrm{CDCl}_{3}, 400 \mathrm{MHz}\right): \delta 8.20(\mathrm{~d}, 2 \mathrm{H}$, $J=10.48, \mathrm{CH}), 7.98(\mathrm{~d}, 2 \mathrm{H}, J=9.36, \mathrm{CH}), 7.82-7.77$ (m, 4H, CH-Ar), 7.62 (t, 2H, $J=1.52,4.64, \mathrm{CH}), 7.59$ (s, 1H, Ar-CH), 7.52 (d, 2H, $J=3.68, \mathrm{CH}-\mathrm{Ar}), 7.43$ (t, 1H, $J=5.24,5.08, \mathrm{CH}-\mathrm{Ar}), 4.01(\mathrm{~s}, 1 \mathrm{H}, \mathrm{NH}), 3.37$ (t, 2H, $J=0.52,6.96, \mathrm{CH}), 2.58-2.54$ (p, 2H, CH) ppm. MS (m/z): $480(\mathrm{M}+1)$.

MPP-11, N-(3-(4-(2-chlorophenyl)-1H-1,2,3-triazol1-yl)propyl)acridin-9-amine, yield: $83 \%$, MP: $242^{\circ}$, $\mathrm{R}_{\mathrm{f}}$ : 0.62; FT-IR (KBr): $\mathrm{cm}^{-1} 3374-\mathrm{N}-\mathrm{H}$ str. (secondary amine), 3031-C-H str. (triazole), 1449-C-H str. (methylene), 1647-C $=\mathrm{N}$ str., $1513-\mathrm{C}=\mathrm{C}$ str. (Ar), 1338$\mathrm{C}-\mathrm{N}$ str., 794-C-Cl str.; ${ }^{1} \mathrm{H}$ NMR $\left(\mathrm{CDCl}_{3}, 400 \mathrm{MHz}\right)$ : $\delta 8.14(\mathrm{~d}, 2 \mathrm{H}, J=7.48, \mathrm{CH}), 7.97(\mathrm{~d}, 2 \mathrm{H}, J=7.24, \mathrm{CH})$, 7.79 (t, 2H, $J=1.92,3.96, \mathrm{CH}), 7.73(\mathrm{~d}, 1 \mathrm{H}, J=6.52$, $\mathrm{CH}), 7.62$ (t, 2H, $J=2.88,5.64, \mathrm{CH}), 7.59$ (s, 1H, Ar-
$\mathrm{CH}), 7.57$ (d, 1H, $J=7.0, \mathrm{CH}-\mathrm{Ar}), 7.41$ (t, $1 \mathrm{H}, J=6.64$, 2.92, CH-Ar), 7.36 (t, 1H, $J=4.56,4.32, \mathrm{CH}-\mathrm{Ar}), 4.48$ (t, 2H, $J=4.24,4.72, \mathrm{CH}-\mathrm{Ar}), 4.01$ (s, 1H, NH), 3.36 (t, 2H, $J=3.36,3.96, \mathrm{CH}), 2.57-2.53$ (p, 2H, CH) ppm. $\operatorname{MS}(\mathrm{m} / \mathrm{z}): 414(\mathrm{M}+1)$.

MPP-12, N-(3-(4-(o-tolyl)-1H-1,2,3-triazol-1-yl) propyl)acridin-9-amine, yield: $88 \%$, MP: $252^{\circ}, \mathrm{R}_{\mathrm{f}}: 0.57$; FT-IR (KBr): $\mathrm{cm}^{-1} 3332-\mathrm{N}-\mathrm{H}$ str. (secondary amine), 3082-C-H str. (triazole), 1463-C-H str. (methylene), 1401-C-H str. (methyl), 1683-C=N str., $1579-\mathrm{N}=\mathrm{N}$ str., $1544-\mathrm{C}=\mathrm{C}$ str. $(\mathrm{Ar}), 1266-\mathrm{C}-\mathrm{N}$ str.; ${ }^{1} \mathrm{H}$ NMR $\left(\mathrm{CDCl}_{3}\right.$, $400 \mathrm{MHz}): \delta 8.20(\mathrm{~d}, 2 \mathrm{H}, J=5.68, \mathrm{CH}), 7.98(\mathrm{~d}, 2 \mathrm{H}$, $J=4.84, \mathrm{CH}), 7.79(\mathrm{t}, 2 \mathrm{H}, J=4.32,3.92, \mathrm{CH}), 7.64$ (t, 2H, $J=3.48,6.2, \mathrm{CH}), 7.59$ (s, 1H, Ar-CH), 7.58 (d, 1H, $J=8.32$, CH-Ar), 7.33-7.28 (m, 3H, CH-Ar), 4.48 (t, 2H, $J=5.0,3.32, \mathrm{CH}), 4.01(\mathrm{~s}, 1 \mathrm{H}, \mathrm{NH}), 3.36$ (t, $2 \mathrm{H}, J=5.36,1.4, \mathrm{CH}), 2.59\left(\mathrm{~s}, 3 \mathrm{H}, \mathrm{CH}_{3}\right), 2.57-2.53$ (p, 2H, CH) ppm. MS (m/z): $394(\mathrm{M}+1)$.

\section{Biological evaluation:}

All newly synthesized triazolyl-acridines were assayed in vitro for cytotoxic activity against MCF-7 (human breast adenocarcinoma cell line) and HT-29 (human colon adenocarcinoma cell line) cells, which were maintained at $37^{\circ}$ in a humidified atmosphere (90\%) containing $5 \% \mathrm{CO}_{2}{ }^{[24]}$.

\section{MTT Assay:}

All the synthesized triazolyl-acridine compounds were dissolved in DMSO and serially diluted with complete medium to get a range of test concentrations. DMSO concentration was kept $<0.1 \%$ in all the samples. HT-29 colon carcinoma and MCF7 cells breast adenocarcinoma maintained in appropriate conditions were seeded in 96 well plates and treated with different concentrations of the test samples and incubated at $37^{\circ}$, $5 \% \mathrm{CO}_{2}$ for $96 \mathrm{~h}$. MTT (3-(4,5-dimethylthiazol-2-yl)2,5-diphenyltetrazoliumbromide) reagent was added to the wells and incubated for $4 \mathrm{~h}$; the dark blue formazan product formed by the cells was dissolved in DMSO under a safety cabinet and read at $550 \mathrm{~nm}$ in triplicate. Percent inhibitions were calculated and plotted with the concentrations used to calculate the inhibitory concentration $\left(\mathrm{IC}_{50}\right)$ values ${ }^{[25]}$. The results of MTT assay summarized in Tables 1 and 2 .

\section{RESULTS AND DISCUSSION}

Structures of novel synthesized triazolyl-acridine compounds, MPP-1 to MPP-12 were confirmed by FT-IR, ${ }^{1} \mathrm{HNMR}$ and mass spectroscopy data as well 
www.ijpsonline.com

TABLE 1: CYTOTOXIC ACTIVITY OF SYNTHESIZED COMPOUNDS AGAINST MCF-7 CELL LINE

\begin{tabular}{|c|c|c|c|c|c|c|}
\hline \multirow{2}{*}{ Compound code } & \multicolumn{5}{|c|}{ Concentration $(\mu \mathrm{M})$} & \multirow{2}{*}{$\begin{array}{c}\text { IC50 value } \\
(\mu \mathrm{M})\end{array}$} \\
\hline & 10 & 1 & 0.1 & 0.01 & 0.001 & \\
\hline MPP-1 & $74.3 \pm 0.23$ & $42.55 \pm 0.00$ & $23.66 \pm 0.04$ & $14.29 \pm 0.08$ & $10.31 \pm 0.01$ & 2 \\
\hline MPP-2 & $55.48 \pm 0.71$ & $43.26 \pm 0.82$ & $29.21 \pm 1.07$ & $7.54 \pm 0.23$ & $1.22 \pm 0.09$ & 2 \\
\hline MPP-3 & $68.36 \pm 1.58$ & $50.1 \pm 2.55$ & $13.24 \pm 0.45$ & $1.26 \pm 0.14$ & $2.3 \pm 0.00$ & $>10$ \\
\hline MPP-4 & $56.64 \pm 1.59$ & $33.64 \pm 0.22$ & $18.54 \pm 1.49$ & $12.35 \pm 0.92$ & $3.69 \pm 0.27$ & $>10$ \\
\hline MPP-5 & $56.64 \pm 0.86$ & $33.21 \pm 0.59$ & $21.54 \pm 0.38$ & $2.38 \pm 0.91$ & $1.25 \pm 0.53$ & 4 \\
\hline MPP-6 & $58.95 \pm 0.17$ & $45.56 \pm 0.24$ & $12.54 \pm 0.21$ & $3.36 \pm 0.09$ & $2.05 \pm 0.09$ & 10 \\
\hline MPP-7 & $41.26 \pm 0.47$ & $32.35 \pm 1.16$ & $23.64 \pm 0.39$ & $10.11 \pm 0.73$ & $2.37 \pm 0.38$ & $>10$ \\
\hline MPP-8 & $38.65 \pm 0.80$ & $28.66 \pm 0.33$ & $12.64 \pm 0.13$ & $10.56 \pm 0.22$ & $2.3 \pm 0.21$ & $>10$ \\
\hline MPP-9 & $68.1 \pm 0.27$ & $50.2 \pm 0.69$ & $32.15 \pm 0.98$ & $13.65 \pm 0.44$ & $10.1 \pm 0.46$ & 1 \\
\hline MPP-10 & $32.15 \pm 0.09$ & $16.54 \pm 0.24$ & $10.2 \pm 0.09$ & $2.5 \pm 0.12$ & $1.1 \pm 0.25$ & $>10$ \\
\hline MPP-11 & $31.21 \pm 0.09$ & $23.5 \pm 0.30$ & $12.3 \pm 0.39$ & $6.27 \pm 0.59$ & $2.13 \pm 0.10$ & $>10$ \\
\hline MPP-12 & $39.21 \pm 0.19$ & $25.32 \pm 0.59$ & $13.5 \pm 0.20$ & $8.24 \pm 0.00$ & $1.34 \pm 0.08$ & $>10$ \\
\hline
\end{tabular}

Results expressed as mean \pm SD of $\%$ inhibition, $\mathrm{IC}_{50}$ values calculated from MTT assay results

TABLE 2: CYTOTOXIC ACTIVITY OF SYNTHESIZED COMPOUNDS AGAINST HT-29 CELL LINE

\begin{tabular}{|c|c|c|c|c|c|c|}
\hline \multirow{2}{*}{ Compound code } & \multicolumn{5}{|c|}{ Concentrations $(\mu \mathrm{M})$} & \multirow{2}{*}{ - IC50 value $(\mu \mathrm{M})$} \\
\hline & 10 & 1 & 0.1 & 0.01 & 0.001 & \\
\hline MPP-1 & $72.3 \pm 0.13$ & $44.22 \pm 0.74$ & $32.7 \pm 0.06$ & $15.74 \pm 0.63$ & $11.2 \pm 0.12$ & 2 \\
\hline MPP-2 & $58.17 \pm 0.18$ & $44.6 \pm 0.12$ & $32.91 \pm 1.26$ & $10.8 \pm 0.01$ & $2.47 \pm 0.29$ & 2 \\
\hline MPP-3 & $32.87 \pm 0.55$ & $15.66 \pm 0.21$ & $12.74 \pm 0.81$ & $2.47 \pm 0.21$ & $1.45 \pm 0.28$ & $>10$ \\
\hline MPP-4 & $52.7 \pm 0.47$ & $32.84 \pm 0.46$ & $22.85 \pm 0.33$ & $18.19 \pm 0.72$ & $2.47 \pm 0.32$ & $>10$ \\
\hline MPP-5 & $53.44 \pm 0.23$ & $37.6 \pm 0.35$ & $22.5 \pm 0.17$ & $7.24 \pm 0.18$ & $3.54 \pm 0.45$ & 4 \\
\hline MPP-6 & $54.25 \pm 0.42$ & $22.61 \pm 0.2$ & $18.4 \pm 0.18$ & $1.2 \pm 0.07$ & $1.1 \pm 0.12$ & 10 \\
\hline MPP-7 & $45.26 \pm 0.01$ & $25.63 \pm 0.78$ & $18.54 \pm 0.37$ & $12.31 \pm 0.35$ & $1.25 \pm 0.16$ & $>10$ \\
\hline MPP-8 & $38.65 \pm 0.32$ & $28.66 \pm 0.28$ & $12.64 \pm 0.10$ & $10.56 \pm 0.33$ & $2.3 \pm 0.16$ & $>10$ \\
\hline MPP-9 & $55.3 \pm 0.63$ & $44.21 \pm 0.24$ & $32.26 \pm 0.52$ & $15.6 \pm 0.36$ & $2.36 \pm 0.21$ & 2 \\
\hline MPP-10 & $45.26 \pm 0.44$ & $32.15 \pm 0.41$ & $21.26 \pm 0.62$ & $6.35 \pm 0.42$ & $1.25 \pm 0.32$ & $>10$ \\
\hline MPP-11 & $42.3 \pm 0.12$ & $22.21 \pm 0.36$ & $12.42 \pm 0.36$ & $6.12 \pm 0.41$ & $2.31 \pm 0.01$ & $>10$ \\
\hline MPP-12 & $32.3 \pm 0.37$ & $20.1 \pm 0.56$ & $11.21 \pm 0.89$ & $4.91 \pm 0.71$ & $1.22 \pm 0.56$ & $>10$ \\
\hline
\end{tabular}

Results expressed as mean \pm SD of \% inhibition, $\mathrm{IC}_{50}$ values calculated from MTT assay results

as their distinct $\mathrm{R}_{\mathrm{f}}$ values in TLC analysis. FT-IR spectroscopic data clearly confirmed the formation of target compounds, by showing stretching of triazole's $\mathrm{C}-\mathrm{H}$ bond at $3000 \mathrm{~cm}^{-1}$, methylene's $\mathrm{C}-\mathrm{H}$ bond about $1465 \mathrm{~cm}^{-1}$, aryl alkyl ether's C-O between 1200-1275 $\mathrm{cm}^{-1}$ (MPP-1 to MPP-6 compounds), secondary amine's N-H bond between 3310-3350 $\mathrm{cm}^{-1}$ (MPP-7 to MPP-12 compounds) and disappearance of azide's peaks found between $2120-2160 \mathrm{~cm}^{-1}$. Similarly proton NMR signal characterized the target compounds by $\delta$-value of, $\mathrm{CH}$ at triazole ring at $7.59 \mathrm{ppm}, \mathrm{CH}_{2}$ ranges $2.1-5.5 \mathrm{ppm}$ and $\mathrm{NH}$ at $4.01 \mathrm{ppm}$ (compound MPP-7 to compounds MPP-12).

All synthesized triazolyl-acridine compounds (MPP1 to MPP-12) were evaluated in vitro for cytotoxicity against MCF-7 and HT-29. IC $_{50}$ values were calculated by MTT assay, using five different concentrations of all triazolyl-acridine compounds. MCF-7 cell line was found highly sensitive against methyl substituted compound (MPP-9) with an $\mathrm{IC}_{50}$ of $1 \mu \mathrm{M}$, unsubstituted compound (MPP-1) and chlorine substituted compounds (MPP-2, MPP-5) exhibited $\mathrm{IC}_{50}$ of $2,4 \mu \mathrm{M}$, respectively. HT-29 cell line was found sensitive with $\mathrm{IC}_{50}$ of $2 \mu \mathrm{M}$ against MPP-1, MPP-2, MPP-9 and MPP5 with $\mathrm{IC}_{50}$ of $4 \mu \mathrm{M}$. The data clearly showed inhibition of growth of MCF-7 and HT-29 cells, which decreased with increasing doses administered. At the final dose, there was only minimal amount of cells survived.

Present study concluded that novel synthesized triazolyl-acridine compounds inhibited the growth of cells tested in a dose-dependent manner. MPP-1, MPP2 and MPP-9 demonstrated greater activity while MPP5 was moderately active in inhibiting the growth of both cell line tested. These can still be considered good candidates for the development of anticancer drugs since these demonstrated activities against cancer cells. These compounds also can be used as drug leads for the development of better candidate drugs or these can be used in combination with other antineoplastic drugs to complement their therapeutic effects. However, more in vitro and in vivo mechanistic studies are required to understand the full potential of these compounds. 


\section{Acknowledgements:}

The authors thank the IKG Punjab Technical University, Kapurthala, Jalandhar where the author is registered as a research scholar. The author also thanks Deshpande Laboratories Pvt. Ltd. Bhopal for evaluating the synthesized compounds for cytotoxic studies and sophisticated analytical instruments facility, Panjab University, Chandigarh for ${ }^{1} \mathrm{H}-\mathrm{NMR}$, IR and Mass spectroscopic data.

\section{Conflict of interest:}

Nil.

\section{Financial support/scholarship:}

Nil.

\section{REFERENCES}

1. Mohammad AA. Phytochemicals as Potential Anticancer Drugs: Time to Ponder Nature's Bounty. BioMed Res Int 2020;7:8602879

2. Hahn W.C. Cancer: Surviving on the Edge. Cancer Cell 2004;6:215-22.

3. Rupar SJ, Dobricic V, Aleksic MM, Brboric SJ, Cudina O. A review of published data on acridine derivatives with different biological activities. Kragujevac J Sci 2018;40:83-101.

4. Gao H, Denny WA, Garg R, Hansch C. Quantitative structureactivity relationships (QSAR) for 9-anilino- acridines: a comparative analysis. Chem Biol Interaction 1998;116:157-80.

5. Lerman LS. The structure of the deoxyribonucleic acid (DNA)-acridine complex. Proc Nat Acad Sci USA 1963;49: 94-102.

6. Demeunynck M. Antitumour Acridines. Expert Opin 2004; 14:55-70.

7. Belmont P, Dorange I. Acridine: A Simple Scaffold with a Wide Range of Application in Oncology. Expert Opin 2008;18:121124.

8. Su TL, Lin YW, Chou TC, Zhang X, Bacherikov VA, Chen $\mathrm{CH}$, et al. Potent Antitumor 9-Anilinoacridines and Acridines Bearing an Alkylating N-Mustard Residue on the AcridineChromophore. Synthesis and Biological Activity. J Med Chem 2006;49:3710-18.

9. Chandra T, Garg N, Lata S, Saxena KK, Kumar A. Synthesis of substituted acridinylpyrazoline derivatives and their evaluation for anti-inflammatory activity. Eur J Med Chem 2010;45:1772-76.

10. Rahimizadeh M, Pordel M, Bakavoli M, Bakhtiarpoor Z, Orafaie A. Synthesis of imidazo [4,5-a]acridones and imidazo [4,5-a] acridines as potential antibacterial agents. Monatsh Chem 2009;140:633-38.

11. Valdes AFC. Acridine and Acridinones: Old and New Structures with Antimalarial Activity. Open Med Chem J 2011;5:11-20.
12. Goodell JR, Madhok AA, Hiasa H, Ferguson DM. Synthesis and evaluation of acridine- and acridone-based anti-herpes agents with topoisomerase activity. Bioorg Med Chem 2006;14:5467-80.

13. Praveena KSS, Murthy NYS, Pal S. Synthesis and biological activities of 1,4-disubstituted-1,2,3-triazoles. J Chem Pharm Res 2015;7:506-22;

14. Reddy PB, Agrawal SK, Singh S, Bhat BA, Saxena AK, Kumar HMS, et al. Synthesis and Biological Evaluation of 4b-[(4Substituted)-1,2,3-triazol-1-yl] podophyllotoxins as Potential Anticancer Agents. Chem Biodivers 2008;5:1792-1802.

15. Jordao AK, Sathler PC, Ferreira VF, Campos VR, de Souza $\mathrm{M}$, Castro HC, et al. Synthesis, antitubercular activity, and SAR study of N-substituted-phenylamino-5-methyl-1H-1,2,3triazole-4-carbohydrazides. Bioorg Med Chem 2011;19:560511.

16. Bengtsson C, EG Lindgren A, Uvell H, Almqvist F. Design, synthesis and evaluation of triazole functionalized ringfused 2-pyridones as antibacterial agents. Eur J Med Chem 2012;54:637-46.

17. Haider S, Alam MS, Hamid H, Shafi S, Nargotra A, Mahajan P, et al. Synthesis of novel 1,2,3-triazole based benzoxazolinones: Their TNF-a based molecular docking with in-vivo antiinflammatory, antinociceptive activities and ulcerogenic risk evaluation. Eur J Med Chem 2013;70:579-88.

18. Garudachari B, Isloor AM, Satyanarayana MN, Fun HK, Hegde G. Click chemistry approach: Regioselective onepot synthesis of some new 8-trifluoromethylquinoline based 1,2,3-triazoles as potent antimicrobial agents. Eur J Med Chem 2014;74:324-32.

19. Piotrowska DG, Balzarini J, Glowacka IE. Design, synthesis, antiviral and cytostatic evaluation of novel isoxazolidine nucleotide analogues with a 1,2,3-triazole linker. Eur J Med Chem 2012;47:501-09.

20. Ullmann, F. On a new formation of diphenylamine derivatives. [machine translation]. Ber Dtsch Chem Ges 1903;36:2382-84.

21. Jayakumar KN, John NA, Kimberly Y, Kekeli A. Ekoue-Kovi, Leah B, et al. 4-N-, 4-S-, and 4-O-Chloroquine Analogues: Influence of Side Chain Length and Quinolyl Nitrogen pKaon Activity vsChloroquine Resistant Malaria. J Med Chem 2008;51:3466-79.

22. Rokhum L, Bez G. A practical one-pot synthesis of azides directly from alcohols. J Chem Sci 2012;124(3):687-91.

23. Fahmi H, Timothy L, Robert H, Vsevolod V. Rostovtsev, Louis $\mathrm{N}$, et al. Copper(I)-Catalyzed Synthesis of Azoles. DFT Study Predicts Unprecedented Reactivity and Intermediates. J Am Chem Soc 2005;127:210-16

24. Van de LAA, Beelen RH, Ossenkoppele GJ, Broekhoven MG, Langenhuijsen MM. A tetrazolium-based colorimetric MTT assay to quantitate human monocyte mediated cytotoxicity against leukemic cells from cell lines and patients with acute myeloid leukemia. J Immunol Methods 1994;174:311-20.

25. Slater TF, Sawyer B, Straeuli U. Studies on succinatetetrazoliumreductase systems. Iii Points of Coupling of Four Different Tetrazolium Salts. Biochim Biophys Acta 1963;77:383-93. 